\title{
One size does not fit all: Nuances in postoperative N2 non-small cell lung cancer management
}

Tim J. Kruser, MD, ${ }^{\mathrm{a}, \mathrm{b}}$ Nisha Mohindra, MD, ${ }^{\mathrm{b}, \mathrm{c}}$ and Malcolm M. DeCamp, MD ${ }^{\mathrm{b}, \mathrm{d}}$

\author{
From the ${ }^{\mathrm{a}}$ Department of Radiation Oncology, ${ }^{\mathrm{c}}$ Division of Hematology and Oncology, Department of Internal \\ Medicine, and ${ }^{\mathrm{d}}$ Division of Thoracic Surgery, Department of Surgery, Feinberg School of Medicine, and ${ }^{\mathrm{b}}$ Ro- \\ bert H. Lurie Comprehensive Cancer Center, Northwestern University, Chicago, Ill. \\ Received for publication Jan 25, 2018; accepted for publication Feb 3, 2018; available ahead of print April 19, \\ 2018. \\ Address for reprints: Malcolm M. DeCamp, MD, Division of Thoracic Surgery, Northwestern Memorial Hospital, \\ 676 No Saint Clair St, Suite 650, Chicago, IL 60611 (E-mail: mdecamp@nm.org). \\ J Thorac Cardiovasc Surg 2018;156:370-3 \\ $0022-5223 / \$ 36.00$ \\ Copyright $(2) 2018$ by The American Association for Thoracic Surgery \\ https://doi.org/10.1016/j.jtcvs.2018.02.100
}

Feature Editor's Note-N2 lung cancer is one of the most complex and controversial areas within thoracic oncology. To a large extent, this is because $N 2$ disease is embodied by a spectrum of disease states that are often grouped together in clinical studies. The variability in the biologic outcomes of N2 disease states is highlighted by proposals for the American Joint Commission on Cancer 8th edition staging system, which included consideration, on the basis of differential survival outcomes, of $N$ descriptor subsets such as single-station N2 disease in the absence of N1 disease (N2a1), single-station N2 disease in the presence of $N 1$ disease (N2a2), and multistation N2 disease (N2b). This subgrouping was ultimately not included in the stage classification of non-small cell lung cancer because of the difficulties of accurate assessment during clinical staging. Such heterogeneity of N2 nonsmall cell lung cancer cohorts within clinical studies that inform management guidelines for these patients has often resulted in differences in opinions among the various disciplines of thoracic oncology regarding interpretation of these data and agreement on best available care. In this Feature Expert Opinion article, a multidisciplinary team of authors provides, with remarkable clarity, a balanced view of the common nuances of $\mathrm{N} 2$ disease. This piece is centered on a recently published randomized trial on the treatment of yet another subset of this disease, occult N2 disease discovered after surgical resection. The results of this trial are discussed in the context of the current landscape for treatment of patients with resectable N2 non-small cell lung cancer, and the reader is promised an enhanced understanding of the gradations of this heterogeneous disease.

\section{Bryan M. Burt, MD}

Despite more frequent use of computed tomographic screening in populations at high risk for lung cancer tion, or both?

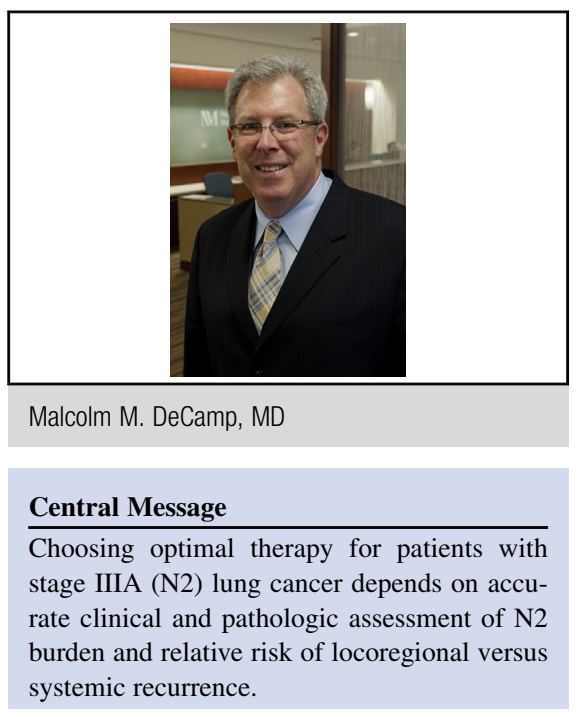

See Editorial Commentary page 374.

development, stage III lung cancer remains a commonly encountered scenario for the practicing thoracic oncologist. Whereas curable stage IIIb disease (7th American Joint Commission on Cancer system) is almost uniformly managed with definitive chemoradiotherapy, the management of stage IIIa disease is widely disparate and continues to be an area of active study and debate. Individual trials involving preselected patients with stage disease IIIa may achieve outcomes that appear promising; however supporting data for the 7th American Joint Commission on Cancer staging manual demonstrates that on a population level, outcomes in stage IIIa remain quite dismal, with 5-year overall survivals (OSs) of $24 \%$ for pathologic stage IIIa disease and $19 \%$ for clinical stage IIIa disease. ${ }^{1}$ These observed prognostic differences between pathologic and clinical stage IIIa disease highlight the fact that stage IIIa disease encompasses a heterogeneous group of patients from both prognostic and therapeutic vantage points.

Although the value of systemic therapy in stage IIIa disease is well established and unquestioned, surgical resection and radiotherapy are both used as locoregional treatment modalities. The main debates in stage IIIa nonsmall cell lung cancer (NSCLC) management revolve around the use and timing of local options-surgery, radia-

A recent randomized study by Sun and colleagues ${ }^{2}$ adds a layer of nuance regarding the use of both surgery and postoperative radiotherapy (PORT) in patients with occult stage 
IIIa (N2) disease who have undergone upfront surgical management. Sun and colleagues ${ }^{2}$ deserve credit for a wellconducted study examining adjuvant chemotherapy alone versus adjuvant concurrent chemoradiotherapy (CCRT) followed by chemotherapy in a subset of patients with stage IIIa (N2) NSCLC - those with "surprise" N2 disease burden. Patients $(n=101)$ were randomly assigned to receive either 4 cycles of adjuvant carboplatin and paclitaxel or CCRT with low-dose cisplatin and paclitaxel with 50 Gy of mediastinal radiotherapy followed by 2 additional cycles of cisplatin plus paclitaxel. The median disease-free survival (primary end point) in the CCRT arm was 24.7 months, not significantly different from the 21.9 months observed in the chemotherapy-alone arm (hazard ratio [HR], 0.94; $P=.40$ ). Likewise, the OS (74.3 months in the CCRT arm and 83.5 months in the chemotherapy arm; HR, 1.33 ; $95 \%$ confidence interval, 0.71-2.49) were not significantly different. These results suggest no benefit to PORT in this narrow subset of patients with stage IIIa (N2) disease. These results hold the potential to influence the approach to patients with pathologic stage IIIa disease; however, they deserve careful and thoughtful review before incorporation into daily clinical practice.

Nodal status has become one of the most important prognostic features in patients with lung cancer and serves as an integral factor in determining optimal treatment approach. ${ }^{3}$ Management of N2 disease remains an area of active debate, and often the therapeutic approach is guided by "how much" N2 disease is present. Currently, in the 7th and 8th editions of the American Joint Commission on Cancer staging manual, lymph node involvement in lung cancer remains categorized according to the location of the metastatic lymph nodes and not according to the number of lymph nodes involved. ${ }^{4}$ Studies suggest, however, that the amount of nodal disease burden (number, bulk, extracapsular extension, or multistation involvement) is a factor of prognostic significance, and it has become a method to subdivide stage III disease. ${ }^{4-6}$ Historically, management of patients with clinical stage IIIa (cN2) NSCLC disease depends on (1) the clinically apparent bulk of nodal disease, (2) the available institutional expertise, and (3) patient factors and preferences. Bulky or multistation $\mathrm{cN} 2$ disease is most commonly managed in a similar fashion to N3 disease, with surgery rarely used in lieu of definitive chemoradiotherapy. An intermediate risk category of "potentially resectable" cN2 disease exists, in which N2 disease is clinically apparent on staging workup, and either definitive chemoradiation or induction therapy after surgery (preferably a lobectomy) can reasonably be entertained. A further subcategory of patients with stage IIIa disease comprises those who have "surprise" N2 disease found on pathologic sampling of removed lymph nodes at the time of lobectomy after thorough preoperative staging of disease as stage I or II.
This clinical scenario is not rare, but it does depend on the rigor of preresectional invasive staging; a recent analysis demonstrated that $22.4 \%$ of patients with resectable NSCLC will have occult lymph node metastases with systematic lymph node inspection at the time of definitive resection.

Despite published guidelines, practices vary in terms of preoperative imaging and mediastinal staging techniques and whether induction therapy is pursued before or after surgery. ${ }^{8,9}$ In the study by Sun and colleagues, ${ }^{2}$ all patients had a positron-emission tomographic scan performed, but only $41 \%$ of patients had any invasive staging performed. This is even more surprising because $27 \%$ had clinical $\mathrm{N} 1$ disease and $65 \%$ had T2 or T3 tumors; both clinical factors are associated with occult $\mathrm{N} 2$ disease and recommended scenarios for invasive staging. . $^{10,11}$

In the groups of patients with surprise N2 disease after surgery and those with resectable clinical N2 disease, PORT has often been recommended to decrease rates of mediastinal recurrence. This practice has not been without controversy, because an often-cited meta-analysis of PORT $^{12}$ examining 10 randomized studies (2232 patients) from 1966 through 1997 highlighted the pitfalls of overuse of PORT. Overall, PORT was associated with a reduction in 2-year survival (from $55 \%$ to $48 \%$ ), with patients with N0 or $\mathrm{N} 1$ disease strongly demonstrating a negative survival impact with PORT. In patients with N2 disease, there was no OS benefit or detriment from PORT. An early Belgian study included in the meta-analysis is instructive. Van Houtte and associates ${ }^{13}$ randomized 224 patients with stage clinical I NSCLC to observation versus 60 Gy PORT delivered to the mediastinum through the (now outdated) cobalt technique. Although PORT decreased locoregional failure from $19 \%$ to $4 \%$, there was an accompanying apparent OS detriment, from $43 \%$ to $24 \%$ at 5 years. Collectively, these studies suggest that the likelihood of mediastinal recurrence must be reasonably high if the benefit of disease control in the mediastinum is to outweigh the detrimental cardiopulmonary effects of PORT and that future investigations of PORT should be limited to the N2 population.

Subsequent studies examining modern radiation delivery in patients with $\mathrm{N} 2$ disease have had a more favorable response to PORT. Three nonrandomized examinations of the National Cancer Database examined PORT in patients with stage IIIa-pN2 disease, and all favored the use of PORT for patients with $\mathrm{pN} 2$ disease. Corso and coworkers ${ }^{14}$ examined 30,552 patients treated from 1998 to 2006 and found by multivariate analysis an association of PORT with improved OS in patients with $\mathrm{pN} 2$ disease (HR, 0.85 ), with a 5 -year OS of $34.1 \%$ versus $27.8 \%$ without PORT. Only $23.8 \%$ of patients with $\mathrm{pN} 2$ disease received PORT during this time frame. Another group ${ }^{15}$ examined a smaller sample of patients from 2004 to 2006 and reached similar conclusions. Robinson and colleagues ${ }^{16}$ examined a 
more recent cohort from 2006 to 2010 . When restricting the analyses to those patients with $\mathrm{pN} 2$ disease who underwent complete resection and received adjuvant chemotherapy, the use of PORT ( $41.2 \%$ of patients) was associated with an improvement in the 5-year OS from $34.8 \%$ to $39.3 \%$ (HR, 0.886; $P=.014$ ).

Although these retrospective studies are prone to selection biases, their findings are supported by a Chinese randomized study by Shen and colleagues, ${ }^{17}$ in which 140 patients with stage IIIa-pN2 disease were randomly assigned to either postoperative chemotherapy alone versus postoperative chemoradiotherapy. Although the study closed early because of low accrual, 140 patients received either 4 cycles of adjuvant cisplatin and paclitaxel, or the same chemotherapy with 50.4 Gy of mediastinal radiotherapy given concurrently with the first 2 cycles of chemotherapy (CCRT arm). The CCRT arm had a median survival of 40 months, versus 28 months in the chemotherapy-alone arm; this difference favoring PORT approached statistical significance (HR, 0.69; $P=.07$ ). The 5-year disease-free survival (DFS) significantly favored the CCRT $(30.3 \%)$ arm relative to the chemotherapy-alone arm $(18.8 \% ; P=.041)$.

How are we to reconcile the randomized study of Shen and colleagues, ${ }^{17}$ which is suggestive of an OS benefit and a significant 5-year DFS benefit to PORT in patients with stage IIIa-pN2 disease, with the randomized study of Sun and colleagues, ${ }^{2}$ which suggested no utility of PORT in patients with stage IIIa-pN2 disease? (Table 1) An examination of the patterns of failures from these 2 studies demonstrates that preoperative staging effectively stratifies the risk of mediastinal recurrence after complete surgical resection. In the study of Sun and colleagues, ${ }^{2}$ which examined patients with "surprise" pN2 disease that had been staged as cN0-N1 with thorough preoperative staging, the locoregional-only recurrence rate was $14.0 \%$ in the chemotherapy-alone arm. As such, most of the patients did not stand to benefit from PORT but were exposed to the associated toxicities. In contrast, the study of Shen and colleagues ${ }^{17}$ did not incorporate preoperative positron-emission tomographic and computed tomographic staging, and in the chemotherapy-alone arm, $49.2 \%$ of patients had locoregional failure after surgical resection.
PORT use reduced this to $27.3 \%$, and this significant improvement in locoregional control translated into the smaller gains in OS and 5-year DFS.

Recent reports have investigated the morbidity and mortality associated with thoracic radiotherapy in a more robust fashion. Wang and associates ${ }^{18}$ reported symptomatic cardiac events in $23 \%$ of patients enrolled in prospective clinical trials delivering dose-escalated definitive thoracic radiotherapy for stage III NSCLC. These events occurred at a median of 26 months after radiotherapy, and significant correlation with cardiac dosimetric parameters was demonstrated. Similarly, a recent analysis of the Radiation Therapy Oncology Group 0617 study examining definitive CCRT showed that higher levels of radiation to the heart are associated with worse OS. ${ }^{19}$ Although these analyses suggest that advanced radiation techniques. such as intensity-modulated radiotherapy. may reduce some of these toxicities, it is vital to not expose patients at low risk of mediastinal recurrence to these potential detrimental effects.

The use of PORT in patients with stage IIIa-pN2 disease therefore deserves careful consideration according to the likelihood of residual mediastinal disease. One criticism of the study of Sun and colleagues ${ }^{2}$ is that it delivered PORT with concurrent low-dose chemotherapy before adequate delivery of full-dose adjuvant chemotherapy. This resulted in $25 \%$ of patients in the CCRT arm not receiving the scheduled full-dose adjuvant chemotherapy cycles after CCRT, clearly a concern in a population in which distant recurrence ( $\sim 40 \%$ in both arms) remains the dominant pattern of recurrence. Current National Comprehensive Cancer Network guidelines recommend that systemic therapy precede PORT ${ }^{10}$; the study of Sun and colleagues ${ }^{2}$ does not fully address the utility of PORT after adequate systemic therapy has been delivered. Further, this study used a carboplatin-based adjuvant chemotherapy regimen, despite lack of evidence for survival benefit with carboplatin in the adjuvant setting. ${ }^{20,21}$ Multiple adjuvant chemotherapy trials in lung cancer have established cisplatin doublets as the optimal strategy for stage II-IIIA disease, and such regimens are endorsed by National Comprehensive Cancer Network, the American Society of Clinical Oncology, and Cancer Care Ontario. ${ }^{22}$ Finally,

TABLE 1. Summary of 2 randomized studies of mediastinal postoperative radiotherapy for stage IIIa-pN2 non-small cell lung cancer

\begin{tabular}{|c|c|c|c|c|c|c|c|c|}
\hline Author, $\mathbf{y}$ & $\mathbf{N}$ & NSCLC patient population & Randomized arm & $\begin{array}{c}\text { Local } \\
\text { relapse }^{*}\end{array}$ & $\begin{array}{l}\text { Median } \\
\text { OS (mo) }\end{array}$ & $P$ value & $\begin{array}{c}\text { Median } \\
\text { DFS (mo) }\end{array}$ & $P$ value \\
\hline \multirow[t]{2}{*}{ Shen et al, ${ }^{17} 2014$} & 135 & All IIIa-pN2 & Adjuvant chemotherapy & $49.3 \%$ & 28 & .073 & 18 & .041 \\
\hline & & & CCRT & $27.3 \%$ & 40 & & 28 & \\
\hline \multirow[t]{2}{*}{ Sun et al, ${ }^{2} 2017$} & 101 & $\begin{array}{l}\text { Clinical stage I-II with } \\
\text { "surprise" pN2 disease }\end{array}$ & Adjuvant chemotherapy & $24 \%$ & 83.5 & .38 & 21.9 & .40 \\
\hline & & & CCRT & $15.7 \%$ & 74.3 & & 24.7 & \\
\hline
\end{tabular}

$N S C L C$, Non-small cell lung cancer; $O S$, overall survival; $D F S$, disease-free survival; $C C R T$, concurrent chemoradiotherapy. *Includes both locoregional-only relapse and simultaneous distant and locoregional relapses. 
the primary end point of DFS should not replace the objective of 5-year OS when pursuing curative intent therapy.

Nonetheless, some important takeaways can be gleaned. First, the predominant pattern of failure for $\mathrm{pN} 2$ disease remains distant. We should therefore redouble our efforts to use invasive staging appropriately at the time of original diagnosis. If $\mathrm{N} 2$ disease can be identified at the time of preresectional staging, then neoadjuvant chemotherapy can be delivered, with the expectation of even better control of systemic micrometastatic disease, while minimizing the risk that chemotherapy delivery is delayed, dose reduced, or compromised by surgical or radiotherapy complications. Patients with clinically apparent N2 disease before surgical resection will likely derive more benefit from PORT than will patients with "surprise" N2 disease. Adjuvant systemic therapy should be prioritized in these "surprise" patients, and it likely should precede if not supplant PORT, rather than be given concurrently. In addition, the ongoing European LUNG ART study ${ }^{23}$ examining PORT in a randomized fashion will shed further light on patient subgroups that may derive benefit from PORT. In the meanwhile, balanced discussions of potential oncologic benefit versus the risk of cardiopulmonary detriment are needed to guide patient-centered decision making.

\section{Conflict of Interest Statement}

Authors have nothing to disclose with regard to commercial support.

\section{References}

1. Detterbeck FC, Boffa DJ, Tanoue LT. The new lung cancer staging system. Chest. 2009; 136:260-71.

2. Sun JM, Noh JM, Oh D, Kim HK, Lee SH, Choi YS, et al. Randomized phase II trial comparing chemoradiotherapy with chemotherapy for completely resected unsuspected N2-positive non-small cell lung cancer. J Thorac Oncol. 2017;12: 1806-13.

3. Rusch VW, Crowley J, Giroux DJ, Goldstraw P, Im JG, Tsuboi M, et al; International Staging Committee; Cancer Research and Biostatistics; Observers to the Committee; Participating Institutions. The IASLC Lung Cancer Staging Project: proposals for the revision of the $\mathrm{N}$ descriptors in the forthcoming seventh edition of the TNM classification for lung cancer. J Thorac Oncol. 2007;2:603-12.

4. Asamura H, Chansky K, Crowley J, Goldstraw P, Rusch VW, Vansteenkiste JF, et al; International Association for the Study of Lung Cancer Staging and Prognostic Factors Committee, Advisory Board Members, and Participating Institutions. The International Association for the Study of Lung Cancer Lung Cancer Staging Project: proposals for the revision of the $\mathrm{N}$ descriptors in the forthcoming 8th edition of the TNM classification for lung cancer. $J$ Thorac Oncol. 2015;10:1675-84.

5. Wei S, Asamura H, Kawachi R, Sakurai H, Watanabe S. Which is the better prognostic factor for resected non-small cell lung cancer: the number of metastatic lymph nodes or the currently used nodal stage classification? J Thorac Oncol. 2011;6:310-8.

6. Saji H, Tsuboi M, Shimada Y, Kato Y, Yoshida K, Nomura M, et al. A proposal for combination of total number and anatomical location of involved lymph no- des for nodal classification in non-small cell lung cancer. Chest. 2013;143: 1618-25.

7. Rusch VW, Hawes D, Decker PA, Martin SE, Abati A, Landreneau RJ, et al. Occult metastases in lymph nodes predict survival in resectable non-small-cell lung cancer: report of the ACOSOG Z0040 trial. J Clin Oncol. 2011;29:4313-9.

8. Rocco G, Nason K, Brunelli A, Varela G, Waddell T, Jones DR. Management of stage IIIA (N2) non-small-cell lung cancer: a transatlantic perspective $\dagger$. Eur $J$ Cardiothorac Surg. 2016;49:1025-7.

9. Veeramachaneni NK, Feins RH, Stephenson BJ, Edwards LJ, Fernandez FG. Management of stage IIIA non-small cell lung cancer by thoracic surgeons in North America. Ann Thorac Surg. 2012;94:922-6; discussion 926-8.

10. National Comprehensive Cancer Network. NCCN clinical practice guidelines in oncology (NCCN Guidelines ${ }^{\circledR}$ ): Non-Small Cell Lung Cancer, version 3.2018February 21, 2018. Available at: https://www.nccn.org/professionals/physician_ gls/pdf/nscl.pdf. Accessed January 22, 2018.

11. Detterbeck FC, Lewis SZ, Diekemper R, Addrizzo-Harris D, Alberts WM. Executive Summary: Diagnosis and management of lung cancer, 3rd ed: American College of Chest Physicians evidence-based clinical practice guidelines. Chest. 2013;143:7S-37S.

12. Burdett S, Stewart L, PORT Meta-analysis Group. Postoperative radiotherapy in non-small-cell lung cancer: update of an individual patient data meta-analysis. Lung Cancer. 2005;47:81-3.

13. Van Houtte P, Rocmans P, Smets P, Goffin JC, Lustman-Maréchal J, Henry J Postoperative radiation therapy in lung caner: a controlled trial after resection of curative design. Int J Radiat Oncol Biol Phys. 1980;6:983-6.

14. Corso CD, Rutter CE, Wilson LD, Kim AW, Decker RH, Husain ZA. Re-evaluation of the role of postoperative radiotherapy and the impact of radiation dose for nonsmall-cell lung cancer using the National Cancer Database. J Thorac Oncol. 2015;10:148-55.

15. Mikell JL, Gillespie TW, Hall WA, Nickleach DC, Liu Y, Lipscomb J, et al. Postoperative radiotherapy is associated with better survival in non-small cell lung cancer with involved N2 lymph nodes: results of an analysis of the National Cancer Data Base. J Thorac Oncol. 2015;10:462-71.

16. Robinson CG, Patel AP, Bradley JD, DeWees T, Waqar SN, Morgensztern D, et al. Postoperative radiotherapy for pathologic N2 non-small-cell lung cancer treated with adjuvant chemotherapy: a review of the National Cancer Data Base. J Clin Oncol. 2015;33:870-6.

17. Shen WY, Ji J, Zuo YS, Pu J, Xu YM, Zong CD, et al. Comparison of efficacy for postoperative chemotherapy and concurrent radiochemotherapy in patients with IIIA-pN2 non-small cell lung cancer: an early closed randomized controlled trial. Radiother Oncol. 2014;110:120-5.

18. Wang K, Eblan MJ, Deal AM, Lipner M, Zagar TM, Wang Y, et al. Cardiac toxicity After radiotherapy for stage III non-small-cell lung cancer: pooled analysis of dose-escalation trials delivering 70 to 90 Gy. J Clin Oncol. 2017;35: 1387-94.

19. Chun SG, Hu C, Choy H, Komaki RU, Timmerman RD, Schild SE, et al. Impact of intensity-modulated radiation therapy technique for locally advanced nonsmall-cell lung cancer: a secondary analysis of the NRG Oncology RTOG 0617 Randomized Clinical Trial. J Clin Oncol. 2017;35:56-62.

20. Strauss GM, Herndon JE II, Maddaus MA, Johnstone DW, Johnson EA, Harpole DH, et al. Adjuvant paclitaxel plus carboplatin compared with observation in stage IB non-small-cell lung cancer: CALGB 9633 with the Cancer and Leukemia Group B, Radiation Therapy Oncology Group, and North Central Cancer Treatment Group Study Groups. J Clin Oncol. 2008;26:5043-51.

21. Pignon JP, Tribodet H, Scagliotti GV, Douillard JY, Shepherd FA, Stephens RJ, et al. Lung adjuvant cisplatin evaluation: a pooled analysis by the LACE Collaborative Group. J Clin Oncol. 2008;26:3552-9.

22. Kris MG, Gaspar LE, Chaft JE, Kennedy EB, Azzoli CG, Ellis PM, et al. Adjuvant systemic therapy and adjuvant radiation therapy for stage I to IIIA completely resected non-small-cell lung cancers: American Society of Clinical Oncology/Cancer Care Ontario clinical practice guideline update. J Clin Oncol. 2017;35:2960-74

23. Le Pechoux C, Dunant A. Radiation Therapy in Treating Patients With Non Small Cell Lung Cancer That Has Been Completely Removed by Surgery (LUNG ART). Available at: https://clinicaltrialsgov/ct2/show/NCT00410683. 